\title{
Biodiesel from Plant Resources-Sustainable Solution to Ever Increasing Fuel Oil Demands
}

\author{
Md Enamul Hoque*, Lu Pui Gee \\ University of Nottingham Malaysia Campus, Jalan Broga, Semenyih, Selangor, Malaysia \\ Email: *enamul.hoque@nottingham.edu.my
}

Received December 27, 2012; revised January 28, 2013; accepted February 15, 2013

Copyright (c) 2013 Md Enamul Hoque, Lu Pui Gee. This is an open access article distributed under the Creative Commons Attribution License, which permits unrestricted use, distribution, and reproduction in any medium, provided the original work is properly cited.

\begin{abstract}
The demand for fuel oil is ever increasing with the advance of the modern world, whereas worldwide reserves of fossil oils are diminishing at an alarming rate. However, there exist large stockpiles of vegetable oil feedstocks that could be exploited to produce fuel oil, called biodiesel with the aid of biotechnology. Initially, the biodiesel produced from vegetable oil did not attract much attention because of its high cost. However, the recent increase in petroleum prices and the uncertainties of petroleum availability led to the renewal of interest in biodiesel production from such sustainable resources (i.e., vegetable oil feedstocks). This research focuses on the production of biodiesel from plant resources, and further investigates the influences of key process parameters, such as the molar ratio of methanol to oil, catalyst concentration, reaction temperature, reaction period and stirring speed on the biodiesel yield. This investigation is to determine the optimum process parameters for maximum biodiesel yield. The biodiesel was produced from three vegetable oil feedstocks, namely palm, soybean and sunflower oil via a transesterification process. It was observed that all the process parameters significantly influenced the biodiesel yield. The maximum biodiesel yields for palm, sunflower and soybean oil feedstocks were found to be $87.5 \%, 83.6 \%$ and $80.2 \%$, respectively at optimum condition. The results suggest that through proper optimization of the process parameters the biodiesel yields could be maximized. In conclusion, the production of biodiesel from plant resources would be regarded as a sustainable solution to the ever increasing demand of fuel oils.
\end{abstract}

Keywords: Sustainable Solution; Fuel Oil; Biodiesel; Plant Resource; Biotechnology; Process Parameter

\section{Introduction}

Biodiesel (known as fatty acid methyl ester) is a monoalkyl ester of long chain fatty acids produced from renewable resources such as virgin vegetable oils, waste vegetable oils, animal fats, algae etc. [1-3]. It is an alternative fuel that is used in compression-ignition diesel engine with slight or no modification, and has attracted great attention because of its renewability, better gas emissivity and biodegradability [4]. Biodiesel has a lower volumetric heating values and higher cetane and flash points [5]. Biodiesel can be used in the pure form noted as B100 or as a blend (e.g. 20\% biodiesel and $80 \%$ petroleum diesel). The biodiesel has been regarded as the potential substitute for petroleum diesel as it offers a number of attractive beneficial properties compared to conventional petroleum diesel [6]. For example, the use of biodiesel maintains a balanced carbon dioxide cycle as

\footnotetext{
${ }^{*}$ Corresponding author.
}

it is based on renewable biological materials. Besides, environmentally friendliness due to reduced emissions (carbon monoxide, sulphur, aromatic hydrocarbons and soot particles) during combustion is of another benefit, while it offers non-toxic and complete biodegradabile properties. The production of biodiesel from various sources has been explored by some researchers [7-14].

Direct use of vegetable oil containing high amount of free fatty acids in diesel engines, can cause oil ring sticking, lubricating problems, poor fuel atomization or even prevention of atomization as a result of plugged orifices, poor cold engine start up, and the creation of gum and other deposits [15]. Therefore, a special procedure is required to diminish the free fatty acids in the vegetable oil and thus converts the vegetable oil to biodiesel which has similar properties and performances to that of petroleum diesel. Potential processes for the production of biodiesel include microemulsion, pyrolysis (thermal cracking) and transesterification. Among all 
these, transesterification is considered to be the most practiced process as it is relatively simple and cost-effective. Besides, it also tends to lower the viscosity of the biodiesel and produces glycerol as a by-product which also has commercial value.

The transesterification is a chemical process that involves a number of consecutive reversible reactions between the triglyceride segment of vegetable oil and an alcohol in the presence of a catalyst to produce ester (i.e. biodiesel) and by-product (i.e. glycerin) [16]. The triglyceride is converted stepwise to diglyceride, monoglyceride and finally glycerol, whereby $1 \mathrm{~mol}$ of alkyl ester is removed at each step. The formation of alkyl ester from monoglyceride is believed to be the step that determines the reaction rate since monoglyceride is the most stable intermediate compound [17]. Generally, the alcohols used in the transesterification process include methanol, ethanol, propanol and butanol. However, methanol is widely used because of its cost effectiveness, and favorable physical and chemical nature (shortest chain alcohol) [18]. A catalyst is usually used to enhance the reaction rate and biodiesel conversion. Since the reaction is reversible, excess alcohol is required to shift the equilibrium to the product side. Various types of catalysts such as alkali-based, acid-based or enzymatic are used depending upon the nature of the oil exploited for the biodiesel production. Alkali-based catalysts include sodium hydroxide $(\mathrm{NaOH})$, potassium hydroxide $(\mathrm{KOH})$ etc. Sulfuric acid $\left(\mathrm{H}_{2} \mathrm{SO}_{4}\right)$ and hydrochloric acid (HCI) are usually used to as acid catalysts, while lipases and algaes have been tested as enzymatic catalysts [16]. However, the choice of catalyst depends on the free fatty acids (FFA) content in the feedstock oil. Alkaline-catalyzed transesterification is much faster than acid-catalyzed transesterification and thus most often used [17].

Overall, this study concentrates on the production of biodiesel from various plant (palm, soybean and sunflower oil) feedstocks, and further investigates the influences of key process parameters, such as the molar ratio of methanol to oil, catalyst concentration, reaction temperature, reaction period and stirring speed on the biodiesel yields.

\section{Materials and Methods}

\subsection{Feedstocks and Reactants}

Three types of locally produced (in Malaysia) vegetable oils were used in the experiments as fundamental feedstocks for the production of biodiesel. They were palm oil (obtained from Yee Lee Edible Oils Private Limited), soybean oil (obtained from Yee Lee Edible Oils Private Limited) and sunflower oil (obtained from Econfood Manufacturing (M) Sdn. Bhd). The analytical-grade methanol and potassium hydroxide $(\mathrm{KOH})$ provided by the Chemical Laboratory, University of Nottingham Ma- laysia Campus were used as the prime reactants.

\subsection{Preparation of Potassium Methoxide}

The measured $\mathrm{KOH}$ was dissolved in methanol within a conical flask. The conical flask had been swirled for few minutes until the $\mathrm{KOH}$ was fully dissolved in methanol and hence, the final solution of potassium methoxide was produced. To prevent the potassium methoxide to be exposed to the air the opening of the conical flask was wrapped with aluminum foil. Otherwise, the potassium methoxide may react with air which can lead to the compromise of its reaction with methanol and catalyst.

\subsection{Transesterification Reaction}

The feedstock oil was firstly preheated in a conical flask for 25 minutes to a preheating temperature (e.g. $55^{\circ} \mathrm{C}$ ) by means of a water bath shaker. Potassium methoxide solution was then added to the preheated oil in the flask. The overall solution was heated to the specified temperature for specified time period with continuous stirring at the specified speed for the transesterification process to occur. The transesterification is a chemical process that involves a series of reversible reactions (Figure 1) between a triglyceride (fat/oil) and an alcohol thus producing esters and glycerol [15]. Generally a catalyst is employed to enhance the reaction rate and ultimately the biodiesel yield. Because of the reversible nature of the transesterification reaction an excess amount of alcohol is required to shift the equilibrium to the product side. For this experiment, the transesterification reaction (i.e. biodiesel production) was started with the primarily set process parameters such as methanol to feedstock molar ratio of $6: 1$, catalyst $(\mathrm{KOH})$ concentration of $1.0 \%$, reaction temperature of $65^{\circ} \mathrm{C}$, reaction period of 3 hours and stirring speed of $130 \mathrm{rpm}$ based on another study [19]. The process parameters were then varied over a range (methanol to feedstock molar ratios of 3:1, 6:1, 9:1, 12:1 \& 15:1; catalyst $(\mathrm{KOH})$ concentrations of $0.50 \mathrm{wt} \%, 0.75$ $\mathrm{wt} \%, 1.0 \mathrm{wt} \%, 1.25 \mathrm{wt} \%$ \& $1.50 \mathrm{wt} \%$; reaction temperatures of $55^{\circ} \mathrm{C}, 60^{\circ} \mathrm{C} \& 65^{\circ} \mathrm{C}$, reaction periods of $1 \mathrm{hr}, 2$ hrs, 3 hrs \& 4 hrs, and stirring speeds of $80 \mathrm{rpm}, 100 \mathrm{rpm}$ $\& 130 \mathrm{rpm})$ to investigate the influences of process pa-

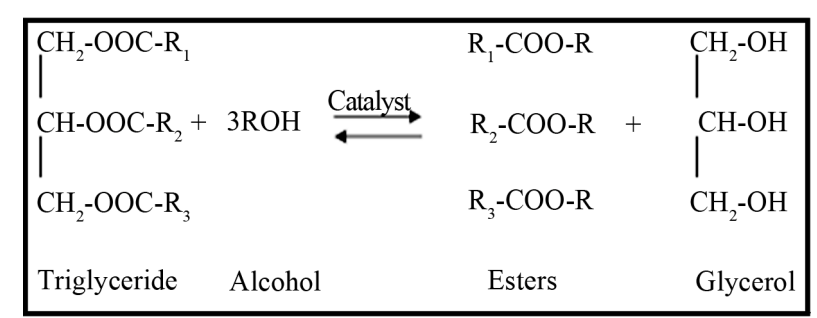

Figure 1. Transesterification process that involves a series of reversible reactions between a triglyceride (fat/oil) and an alcohol which produces esters and glycerol [15]. 
rameters on the biodiesel yields. Upon completion of the transesterification reaction at various set parameters, a mixture of biodiesel and glycerin was produced.

\subsubsection{Separation of Biodiesel}

The hot biodiesel mixture was cooled to room temperature and allowed to settle overnight or at least for a period of 8 hours until the distinct separate layers of biodiesel and glycerin were formed. The biodiesel (golden color) was settled at the top, while the glycerin (dark brown) was at the bottom. The crude biodiesel separated from glycerin by means of a pipette, was taken into another conical flask for washing.

\subsubsection{Washing of Biodiesel}

The crude biodiesel was washed with distilled water to remove impurities like, traces of glycerin, methanol or potassium hydroxide. The traces of glycerin, methanol or potassium hydroxide were absorbed into the water and thus the leaving the biodiesel pure. The water absorbing the impurities turned white murky, which was allowed to settle for an hour for separation from the biodiesel. The biodiesel was settled at the top and the water absorbing impurities was at the bottom. The washing process was repeated until the water became completely clear. At the end the pure biodiesel was extracted by means of a pipette and preserved in a conical flask for further evaluation.

\section{Results and Discussions}

The biodiesel was produced from three vegetable oil feedstocks, namely palm, soybean and sunflower oil via transesterification process with a range of various process parameters, such as molar ratio of methanol to oil, catalyst concentration, reaction temperature, reaction period and stirring speed. The influences of these process parameters on the biodiesel yields are described and discussed as below.

\subsection{Influence of Methanol to Oil Molar Ratio}

In the production of biodiesel from vegetable oils through transesterification process, the methanol/oil molar ratio is observed to play significant role. The transesterification is an equilibrium process and is generally carried out with more alcohol than that required for actual reaction to shift the equilibrium to the expected product (i.e. methyl ester) side. It is reported that the transesterification reaction is inadequate at the methanol/oil molar ratio below 5:1 [20]. Various methanol/oil molar ratios numerically 3:1, 6:1, 9:1, 12:1 and 15:1 were used to investigate the influence of variation of methanol/oil molar ratio on the biodiesel yields as presented in Figure 2. At lower methanol/oil molar ratio (e.g. 3:1) the biodiesel yields were found to be relatively lower for all three feedstocks (palm, sunflower and soybean oils). The biodiesel yields at the methanol/oil molar ratio of $3: 1$ were found to be $70.8 \%, 68.4 \%$ and $65.6 \%$ for palm, sunflower and soybean oils, respectively. The biodiesel yields increased with the increase of methanol/oil molar ratio and reached the highest yields at the molar ratio value of 6:1. The highest biodiesel yields at the methanol/oil molar ratio of $6: 1$ were found to be $87.5 \%, 83.6 \%$ and $80.2 \%$ for palm, sunflower and soybean oils, respectively. Other studies also suggested the

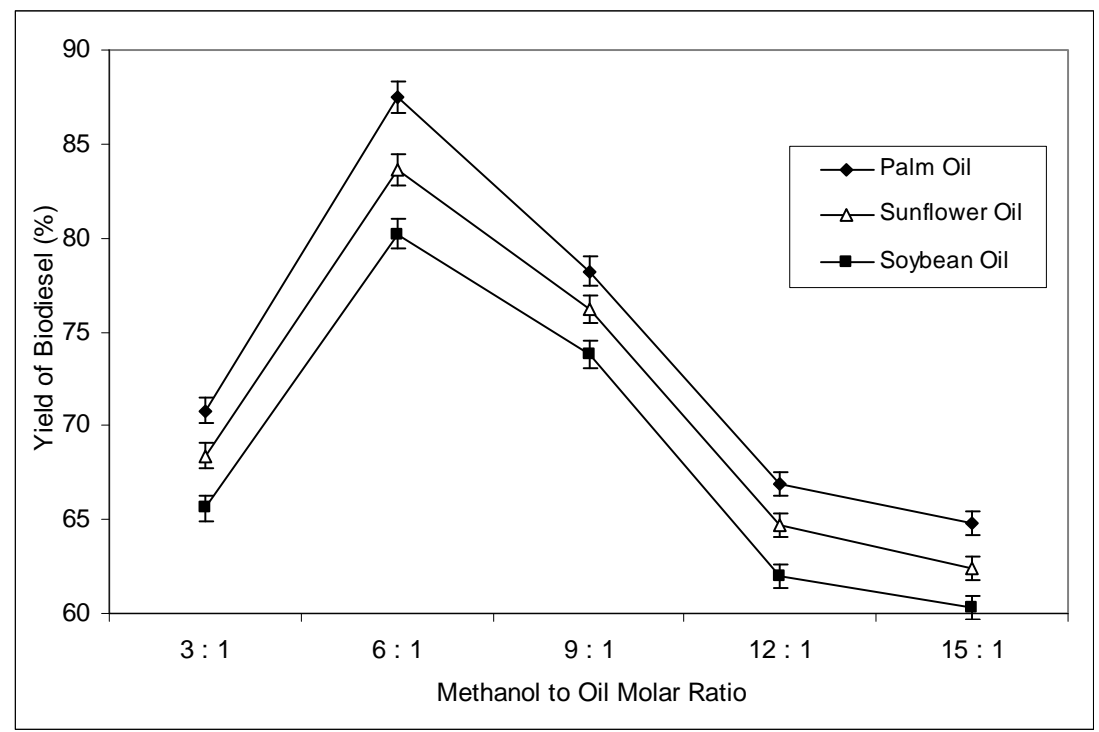

Figure 2. Influence of methanol/oil molar ratio on biodiesel yields for various feedstock oils, with other parameters constant (catalyst $(\mathrm{KOH})$ concentration of $1.0 \%$, reaction temperature of $65^{\circ} \mathrm{C}$, reaction period of 3 hours and stirring speed of 130 rpm). 
optimum molar ratio to be in between 4.8:1 to $6.5: 1$ for the maximum biodiesel yields from other feedstock oils (e.g. animal fat, used cooking oil etc.) [5].

At the molar ratio higher than 6:1 the biodiesel yields were decreased as observed in Figure 2. For example, at the molar ratio of 15:1 the biodiesel yields for palm, sunflower and soybean oils were $68.8 \%, 64.4 \%$ and $63.3 \%$, respectively which had been significantly lower than the highest yields obtained at the molar ratio of 6:1. The decline of biodiesel yields at higher molar ratio than 6:1 could be due to the phenomenon that the excess methanol deactivated the catalyst, thus reducing its reactivity. The excess methanol also caused the biodiesel extraction difficult upon transesterification process by blurring the separation border between produced biodiesel and glycerin. Therefore, the molar ratio of 6:1 was assigned to be the optimum for the biodiesel conversion, and thus this molar ratio (6:1) was kept constant throughout the experiment to evaluate the influences of other parameters on the biodiesel yields. The biodiesel yields were also observed to be varied among the feedstock oils even at the same methanol/oil molar ratio. It could be mainly due to the differences of free fatty acid (FFA) and water contents in the feedstock oils [13], which caused the catalyst to react with the oils variably.

\subsection{Influence of Catalyst Concentration (\% KOH)}

A range of $\mathrm{KOH}$ concentrations, numerically $0.5 \%$, $0.75 \%, 1.00 \%, 1.25 \%$ and $1.50 \%$ weights of oils were used to study the influence of catalyst concentration on the biodiesel yields. The biodiesel yields against various catalyst concentrations are presented in Figure 3. The biodiesel yields significantly increased with the increase of catalyst concentration, producing the highest yields at the $\mathrm{KOH}$ concentration of $1.0 \%$. The highest biodiesel yields for palm, sunflower and soybean oils were found to be $87.5 \%, 83.6 \%$ and $80.2 \%$, respectively. The increased conversion of biodiesel with the increase of catalyst concentration was believed to be due to the increased solubility of methanol into feedstock oils that enhanced biodiesel conversion reaction [19]. The highest yield could be because of maximum ester conversion by the ample amount of catalyst that rendered optimum solubility of methanol into feedstock oil.

Lower catalyst concentration (e.g. $0.5 \% \mathrm{KOH}$ ) resulted in lower biodiesel yields, like 57.8\%, 53.3\% and $49.0 \%$ for palm, sunflower and soybean oils, respectively. It could be due to rather limited biodiesel conversion (i.e. transesterification) because of less influence of catalyst on the solubility of methanol into feedstock oil. Likewise, at higher catalyst (i.e. $\mathrm{KOH}$ ) concentrations (e.g. 1.25\% and $1.50 \%$ ) than $1.0 \%$ the biodiesel yields declined remarkably due to the formation of fatty acid salts (i.e. soap) by saponification process [19]. The produced soap blurred the clear separation of biodiesel from glycerin and also increased the viscosity of the biodiesel, and thus lowering the biodiesel yield. Therefore, $1.0 \% \mathrm{KOH}$ was considered to be the optimum catalyst concentration and utilized for further investigations.

\subsection{Influence of Reaction Temperature}

The transesterification reaction was conducted at three different temperatures (e.g. $55^{\circ} \mathrm{C}, 60^{\circ} \mathrm{C}$ and $65^{\circ} \mathrm{C}$ ), while other parameters were maintained constant like, methanol/oil molar ratio of $6: 1$, catalyst concentration of $1.0 \%$

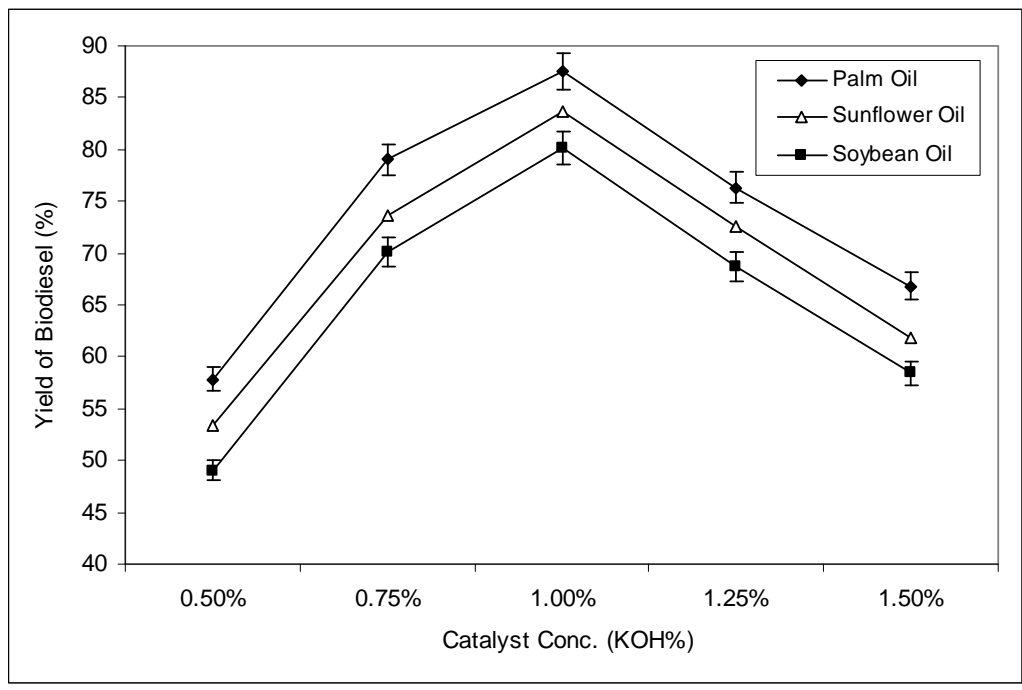

Figure 3. Influence of catalyst concentration $(\% \mathrm{KOH})$ on biodiesel yields for various feedstock oils, with other parameters constant (methanol/oil molar ratio of 6:1, reaction temperature of $65^{\circ} \mathrm{C}$, reaction period of 3 hours and stirring speed of 130 rpm). 
$\mathrm{KOH}$, reaction period of 3 hours and stirring speed of $130 \mathrm{rpm}$. Although the biodiesel conversion (i.e. transesterification) process can happen at a range of temperatures (e.g. from ambient to a temperature close to the boiling point of methanol) usually higher temperature speeds up the reaction rate and shortens the reaction period. The maximum reaction temperature was set at $65^{\circ} \mathrm{C}$ with the consideration that the temperature higher than $65^{\circ} \mathrm{C}$ might cause burning of methanol. On the other hand, the minimum temperature of $55^{\circ} \mathrm{C}$ was taken into consideration with the assumption that too low temperature would slow down the reaction rate and thus increase the reaction period too long, which might not be technically favorable.

The overall biodiesel yields for different feedstock oils at different reaction temperatures are presented in Table 1. The results show that the biodiesel yields for all feedstocks significantly increase with the increase of temperature as demonstrated in Figure 4. For example, the biodiesel yields at the temperatures of $55^{\circ} \mathrm{C}, 60^{\circ} \mathrm{C}$ and $65^{\circ} \mathrm{C}$ were found to be $81.38 \%, 83.24 \%$ and $87.5 \%$, respectively for palm oil, $78.31 \%, 80.17 \%$ and $83.6 \%$, respectively for sunflower oil, and $76.07 \%, 77.93 \%$ and $80.2 \%$, respectively for soybean oil. The highest yields of $87.5 \%, 83.6 \%$ and $80.2 \%$ were obtained for the palm, sunflower and soybean oil feedstocks, respectively at highest reaction temperature of $65^{\circ} \mathrm{C}$. This could be attributed to the phenomenon that the higher temperature (means higher energy input) increased the collision among the reacting molecules, which accelerated the chemical reaction (i.e. transesterification process) and thus increased the biodiesel yields. However, there had been variation in highest yield for different feedstock oils that could be because of the variation in their fatty acid compositions. Therefore, the reaction temperature of $65^{\circ} \mathrm{C}$ was considered as optimal and was maintained for evaluating other parameters.

Some other studies also reported the similar findings that the biodiesel yield was influenced by the reaction temperature [19,21]. In the investigation of the influence of temperature on the biodiesel conversion, National Biodiesel Board [20] observed that the increase of temperature from $30^{\circ} \mathrm{C}$ to $50^{\circ} \mathrm{C}$ increased the biodiesel yield by $10 \%$. Canakci and Gerpen reported that the biodiesel yield had declined at the reaction temperature as high as $70^{\circ} \mathrm{C}$ [22]. They argued that too high temperature promoted the saponification reaction that negatively affected the transesterification process (i.e. biodiesel conversion).

\subsection{Influence of Reaction Period}

Besides the temperature, reaction period also plays significant role in the optimization of biodiesel yield that allows completion of the biodiesel conversion (i.e. transesterification) reaction. Therefore, the transesterification reaction was conducted for the periods of $1,2,3$ and 4 hours maintaining other parameters constant (oil/methanol molar ratio of $6: 1$, catalyst concentration of $1.0 \%$ weight of oil, reaction temperature of $65^{\circ} \mathrm{C}$ and stirring speed of $130 \mathrm{rpm}$ ) to evaluate the influence of reaction period on the biodiesel yields. The overall biodiesel yields for different feedstock oils at different reaction periods are presented in Table 1. The yields were found to be increased significantly with the increase of reaction period reaching maximum values at the reaction period of 3 hours above which the yield values declined as demonstrated in Figure 5. For example, the reaction periods of 1, 2, 3 and 4 hrs resulted in the biodiesel yields of $79.709 \%, 83.98 \%, 87.5 \%$ and $87.5 \%$, respectively for palm oil, $76.1 \%$, $80.56 \%$, $83.6 \%$ and $81.7 \%$, respectively for sunflower oil, and $72.61 \%, 76.03 \%, 80.2 \%$ and $77.7 \%$, respectively for soybean oil. At the reaction pe-

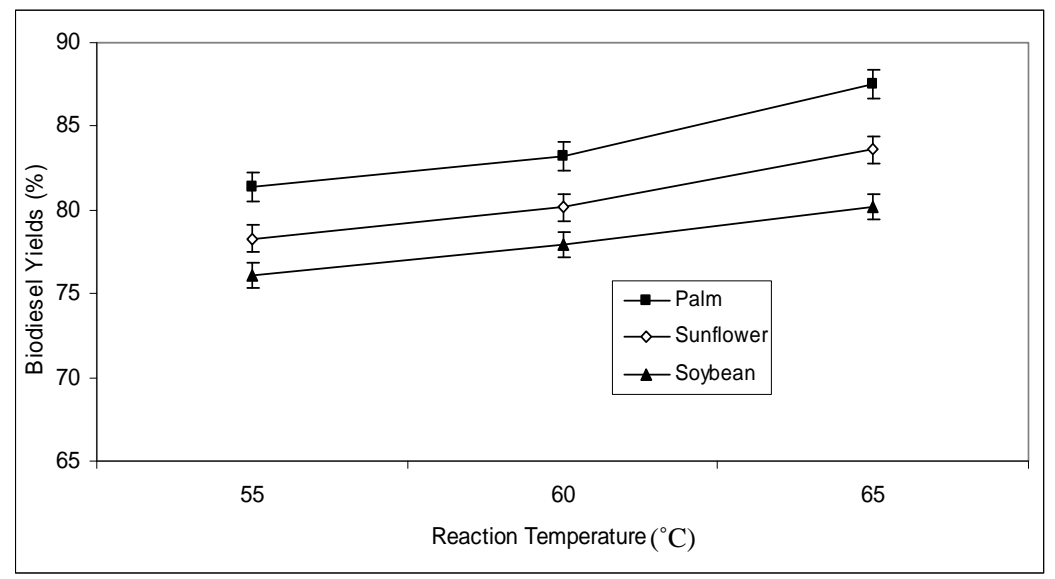

Figure 4. Influence of reaction temperature on biodiesel yields for various feedstock oils, with other parameters constant (methanol/oil molar ratio of $6: 1$, catalyst $(\mathrm{KOH})$ concentration of $1.0 \%$, reaction period of 3 hours and stirring speed of 130 rpm). 


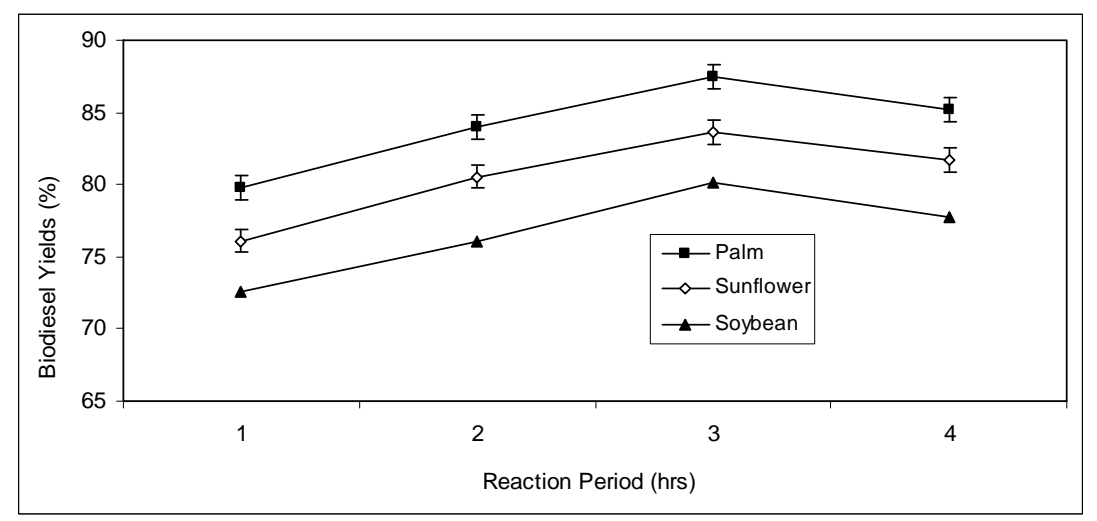

Figure 5. Influence of reaction period on biodiesel yields for various feedstock oils, with other parameters constant (methanol/oil molar ratio of 6:1, catalyst $(\mathrm{KOH})$ concentration of $1.0 \%$, reaction temperature of $65^{\circ} \mathrm{C}$, and stirring speed of 130 rpm).

Table 1. Biodiesel yields at different reaction temperatures and reactions periods for different feedstock oils, with other parameters constant (e.g. methanol to oil molar ratio of 6:1, catalyst (KOH ) concentration of $1.0 \%$, and stirring speed of $130 \mathrm{rpm})$.

\begin{tabular}{|c|c|c|c|}
\hline \multicolumn{4}{|c|}{ Biodiesel Yields at Reaction Temperature of $55^{\circ} \mathrm{C}$} \\
\hline Reaction Period (hr) & Palm Oil & Sunflower Oil & Soybean Oil \\
\hline 1 & 72.819 & 70.773 & 68.727 \\
\hline 2 & 77.376 & 74.679 & 71.61 \\
\hline 3 & 81.375 & 78.306 & 76.074 \\
\hline 4 & 79.515 & 74.493 & 73.377 \\
\hline \multicolumn{4}{|c|}{ Biodiesel Yields at Reaction Temperature of $60^{\circ} \mathrm{C}$} \\
\hline Reaction Period (hr) & Palm Oil & Sunflower Oil & Soybean Oil \\
\hline 1 & 74.679 & 72.633 & 70.587 \\
\hline 2 & 79.236 & 76.539 & 73.47 \\
\hline 3 & 83.235 & 80.166 & 77.934 \\
\hline 4 & 81.375 & 76.353 & 74.679 \\
\hline \multicolumn{4}{|c|}{ Biodiesel Yields at Reaction Temperature of $65^{\circ} \mathrm{C}$} \\
\hline Reaction Period (hr) & Palm Oil & Sunflower Oil & Soybean Oil \\
\hline 1 & 79.79 & 76.1 & 72.61 \\
\hline 2 & 83.98 & 80.56 & 76.03 \\
\hline 3 & 87.5 & 83.6 & 80.2 \\
\hline 4 & 85.21 & 81.7 & 77.7 \\
\hline
\end{tabular}

riod of 3 hours, the obtained highest yields for the palm, sunflower and soybean oil feedstocks were $87.5 \%$, $83.6 \%$ and $80.2 \%$, respectively. The increase of biodiesel yield with the increase of reaction period could be associated with the molecular structure of the oil, which con- tains saturated fatty acids. These fatty acids having higher activation energy require longer period of heating for reaction to occur/complete. It was believed that at the reaction period of 3 hours the required activation energy was achieved giving rise to the equilibrium of the reaction, and thus the biodiesel yield reached maximum value. The prolonged heating might revert back the transesterification reaction direction which led to decline the biodiesel yield after 3 hours. Other study also reported that the reaction period had influenced the yield of biodiesel produced via transesterification process from vegetable oil feedstocks [23].

\subsection{Influence of Stirring Speed}

For the chemical reaction to occur, it is important for the reactants to come into close contact. In the conversion of biodiesel (i.e. transesterification process), the stirring speed was considered to be an important factor that might have influenced the biodiesel yield. The transesterification process was carried out at three different stirring speeds of 80,100 and $130 \mathrm{rpm}$, while other parameters were made to remain constant (e.g. methanol to oil molar ratio of $6: 1$, catalyst concentration of $1.0 \%$, reaction temperature of $65^{\circ} \mathrm{C}$, and reaction period of 3 hours). The influence of stirring speed on the biodiesel yield is presented in Figure 6. It was observed that the biodiesel yields increased as the stirring speed increased for all three feedstock oils. For example, the biodiesel yields at the stirring speeds of 80,100 and $130 \mathrm{rpm}$ were found to be $78.3 \%, 82.2 \%$ and $87.5 \%$, respectively for palm oil, $75.1 \%, 79.3 \%$ and $83.6 \%$, respectively for sunflower oil, and $72.5 \%, 76.4 \%$ and $80.2 \%$, respectively for soybean oil. Higher yield at increased stirring speed was believed to be due to the phenomenon that vigorous agitation/stirring enhanced intimate contact/interaction between the reactant molecules (i.e. oil and $\mathrm{KOH}$ ), which thus resulted in increased biodiesel production. 


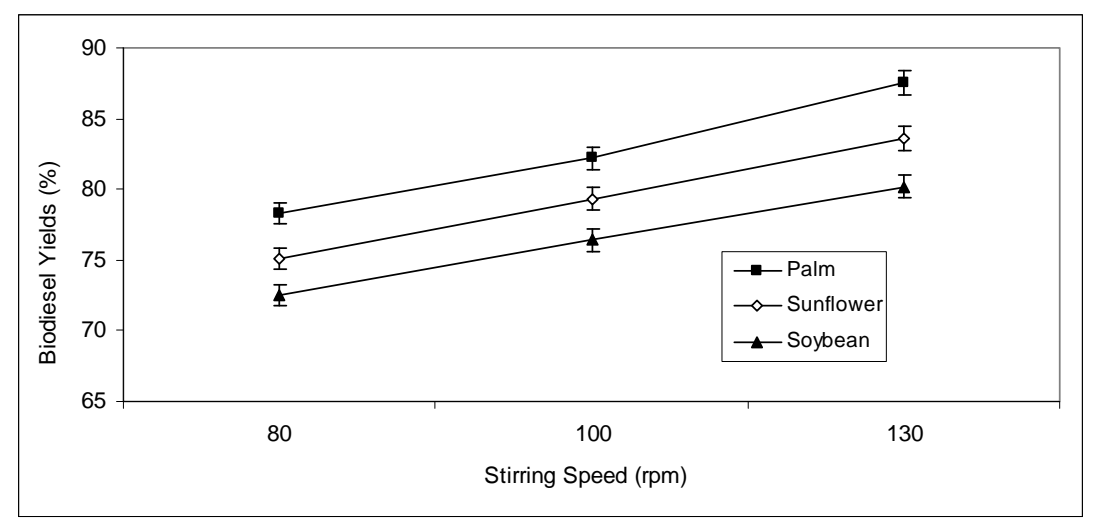

Figure 6. Influence of stirring speed on biodiesel yields for various feedstock oils, with other parameters constant (methanol/oil molar ratio of $6: 1$, catalyst $(\mathrm{KOH})$ concentration of $1.0 \%$, reaction temperature of $65^{\circ} \mathrm{C}$ and reaction period of $3 \mathrm{hrs}$ ).

\section{Conclusion}

Various plant feedstocks (palm, soybean and sunflower oil) were exploited to successfully produce biodiesel via transesterification process which is considered to be more economic in compared to other processes (e.g. supercritical methanol, thermal cracking, microemulsion etc.). Besides the cost effectiveness, transesterification process allows a wide range of parameters to play with for the maximization of biodiesel yield and easy recovery of byproduct glycerin. The process parameters had direct influence on the biodiesel yields for all three types of feedstocks. For maximum biodiesel yields, the optimum parameters were determined to be methanol/oil molar ratio of $6: 1$, catalyst $(\mathrm{KOH})$ concentration of $1.0 \%$, reaction temperature of $65^{\circ} \mathrm{C}$, a reaction period of 3 hours and stirring speed of $130 \mathrm{rpm}$. At optimum condition, the maximum biodiesel yields were measured to/be $87.5 \%$, $83.6 \%$ and $80.2 \%$ of the palm, sunflower and soybean oil feedstocks, respectively. In conclusion, the vegetable oil feedstocks hold high potential as renewable plant resources for the production of biodiesel that is to contribute as a sustainable solution to the ever increasing fuel oil demands. However, the exploitation of vegetable oil feedstocks in producing biodiesel may give arise to another concern (e.g. extensive acreage required for sufficient production of oilseed crops etc.), which should be dealt accordingly.

\section{Acknowledgements}

The authors would like to thank the Faculty of Engineering, University of Nottingham Malaysia Campus for providing the fund to carry out this highly prospective research.

\section{REFERENCES}

[1] R Kalscheuer, T Stolting and A Steinbuchel, "Microdiesel: Escherichia coli Engineered for Fuel Production,”
Microbiology, Vol. 152, No. 9, 2006, pp. 2529-2536. doi:10.1099/mic.0.29028-0

[2] P. M. Schenk, S. R. Thomas-Hall, E. Stephens, U. C. Marx, J. H. Mussgnug, C. Posten, O. Kruse and B. Hankamer, "Second Generation Biofuels: High-Efficiency Microalgae for Biodiesel Production,” Bioenergy Research, Vol. 1, No. 1, 2008, pp. 20-43. doi:10.1007/s12155-008-9008-8

[3] K. M. Weyer, D. R. Bush, A. Darzins and B. D. Willson, “Theoretical Maximum Algal Oil Production,” Bioenergy Research, Vol. 3, No. 2, 2010, pp. 204-213. doi:10.1007/s12155-009-9046-X

[4] T. Prokop, “Imperial Western Products,” In: Communication, Chandler: St., Coachella, 2002, p. 14970.

[5] J. Jitputti, B. Kitiyanan, P. Rangsunvigit, K. Bunyakiat, L. Attanatho and P. Jenvanitpanjakul, "Transesterification of Crude Palm Kernel Oil and Crude Coconut Oil by Different Solid Catalyst," Chemical Engineering Journal, Vol. 116, No. 1, 2006, pp. 61-66. doi:10.1016/j.cej.2005.09.025

[6] T. Krawczyk, "Biodiesel-Alternative Fuel Makes Inroads but Hurdles Remain,” INFORM, Vol. 7, No. 8, 1996, pp. 801-829.

[7] B. Freedman, E. H. Pryde and T. L. Mounts, "Variables Affecting the Yields of Fatty Esters from Transesterified Vegetable Oils," Journal of the American Oil Chemists' Society, Vol. 61, No. 10, 1984, pp. 1638-1643. doi:10.1007/BF02541649

[8] B. Freedman, R. O. Butterfield and E. H. Pryde, "Transesterification Kinetics of Soybean Oil," Journal of the American Oil Chemists' Society, Vol. 63, No. 10, 1986, pp. 1375-1380. doi:10.1007/BF02679606

[9] F. Ma, L. D. Clements and M. A. Hana, “The Effects of Catalyst, Free Fatty Acids and Water on transesterification of Beef Tallow," Transactions of the ASAE (American Society of Agricultural Engineers), Vol. 41, No. 5, 1998, pp. 1261-1264.

[10] M. Canakci and J. V. Gerpen, "Biodiesel Production via Acid Catalysis,” Transactions of the ASAE (American Society of Agricultural Engineers), Vol. 42, No. 5, 1999, pp. 1203-1210. 
[11] M. Iso, B. Chen, M. Eguchi, T. Kudo and S. Shrestha, "Production of Biodiesel Fuel from Triglycerides and Alcohol Using Immobilized Lipase,” Journal of Molecular Catalysis B: Enzymatic, Vol. 16, No. 1, 2001, pp. 5358. doi:10.1016/S1381-1177(01)00045-5

[12] G. Antonlin, F. V. Tinaut, Y. Brceno, V. Castaño, C. Póez and A. I. Ramírez, "Optimisation of Biodiesel Production by Sunflower Oil Transesterification,” Bioresource Technology, Vol. 83, No. 2, 2002, pp. 1111-1114.

[13] A. Demirbas, "Biodiesel Fuels from Vegetable Oils via Catalytic and Non-Catalytic Supercritical Alcohol Transesterifications and Other Methods: A Survey," Energy Conversion and Management, Vol. 44, No. 13, 2003, pp. 2093-2109. doi:10.1016/S0196-8904(02)00234-0

[14] U. Rashid and F. Anwar, "Production of Biodiesel through Optimized Alkaline Catalysed Transesterification of Rapeseed Oil,” Fuel, Vol. 87, No. 3, 2008, pp. 265-273. doi:10.1016/j.fuel.2007.05.003

[15] F. Ma and M. A. Hanna, "Biodiesel Production: A Review,” Bioresource Technology, Vol. 70, No. 1, 1999, pp. 1-15. doi:10.1016/S0960-8524(99)00025-5

[16] J. Marchetti, V. Miguel and A. Errazu, "Possible Methods for Biodiesel Production,” Renewable \& Sustainable Energy Reviews, Vol. 11, No. 6, 2007, pp. 1300-1311. doi:10.1016/j.rser.2005.08.006
[17] M. Cetinkaya and F. Karaosmanoglu, "Optimisation of BaseCatalysed Transesterification Reaction of Used Cooking Oil,” Energy \& Fuels, Vol. 18, No. 6, 2004, pp. 18881895. doi:10.1021/ef049891c

[18] A. Khan, "Research into biodiesel, "Kinetics and Catalyst Development. Individual Inquiry,” University of Queensland, Brisbane, 2002.

[19] M. E. Hoque, A. Singh and Y. L. Chuan, "Biodiesel from Low Cost Feedstocks: The Effects of Process Parameters on the Biodiesel Yield,” Biomass and Bioenergy, Vol. 35, No. 4, 2011, pp. 1582-1587.

[20] National Biodiesel Board, 2009. www.biodieselorg

[21] K. G. Georgogianni, M. G. Kontominas, E. Tegou, D. Avlonitis and V. Vergis, "Biodiesel Production: Reaction and Process Parameters of Alkali-Catalysed Transesterification of Waste Frying-Oils,” Energy \& Fuels, Vol. 21, No. 5, 2007, pp. 3023-3027. doi:10.1021/ef070102b

[22] M. Canakci and J. V. Gerpen, "Biodiesel Production from Oils and Fats with High Free Fatty Acids," Transactions of the ASAE, Vol. 44, No. 6, 2001, pp. 1429-1436.

[23] A. Demirbas, "Biodiesel from Vegetable Oils via Transesterification in Supercritical Methanol,” Energy Conversion and Management, Vol. 43, No. 17, 2002, pp. 23492356. doi:10.1016/S0196-8904(01)00170-4 